\title{
Brucella Epididymo-Orchitis: A Consideration in Endemic Area
}

\author{
Jaffar A. Al-Tawfiq \\ Internal Medicine Services Division, Dhahran Health Center, Saudi Aramco Medical Services \\ Organization, Saudi Aramco, Dhahran, Saudi Arabia
}

\begin{abstract}
Brucellosis is a zoonotic disease caused by Brucella sp. and may affect many parts of the body. Brucella epididymo-orchitis had been reported in up to $20 \%$ of patients with brucellosis. This is a case report of Brucella epididymo-orchitis in a Saudi male patient. He presented with a unilateral swelling of the left testicle. He had fever, arthralgia and night sweats. Ultrasound examination revealed enlarged left epididymis and testicle. Brucella serology was positive and the patient responded to treatment with doxycycline and gentamicin. Thus, brucella infection should be considered in the differential diagnosis of patients presenting with epididymo-orchitis from an endemic area.
\end{abstract}

Key words: epididymitis; orchitis; Brucella; diagnosis; therapeutics

Int Braz. J Urol. 2006; 32: 313-5

\section{INTRODUCTION}

Epididymo-orchitis is usually caused by Neisseria gonorrhea and Chlamydia trachomatis in male patients younger than 35 years of age and by enterobacteriaceae in patients older than 35 years of age. Other causes of epididymo-orchitis include Brucella sp. in endemic areas. Genitourinary system involvement occurs in $2-20 \%$ of patients with brucellosis and includes prostatitis, epididymoorchitis, cystitis, pyelonephritis, interstitial nephritis, exudative glomerulonephritis and renal abscess.

Here we describe a case of Brucella epididymo-orchitis and draw attention to this diagnosis in patients presenting with epididymoorchitis from endemic areas.

\section{CASE REPORT}

A forty-one year old Saudi male presented with a 2 -week history of fever, night sweats, and arthralgia. He had a 2-day history of left testicular swelling but no urinary symptom. He had a history of raw milk ingestion few weeks earlier. His temperature was $39^{\circ} \mathrm{C}$ and systemic examination was unremarkable. The left epididymis and left testicle were enlarged and tender. The white blood cell (WBC) count was $5400 / \mathrm{mm}^{3}$. Blood cultures were negative. Urinalysis showed a WBC of $0-5 /$ high power field (hpf) and red blood cell of 10-25/hpf. Testicular ultrasound revealed an enlarged left testicle measuring $3.4 \times 3.8 \times 4.9 \mathrm{~cm}$ with increased blood flow (Figure1). The left epididymis was enlarged with increased back shadowing raising the possibility of early abscess formation (Figure-2). Brucella abortus antibodies were $<1: 160$ for $\operatorname{IgG}$ and $>1: 2560$ for IgM. The patient was treated with doxycycline (6 weeks) and gentamicin ( 2 weeks) with complete resolution of all signs and symptoms.

\section{COMMENTS}

Brucellosis is a zoonotic disease, which may be caused by 4 Brucella species: B. abortus, B. 


\section{Brucella Epididymo-Orchitis}
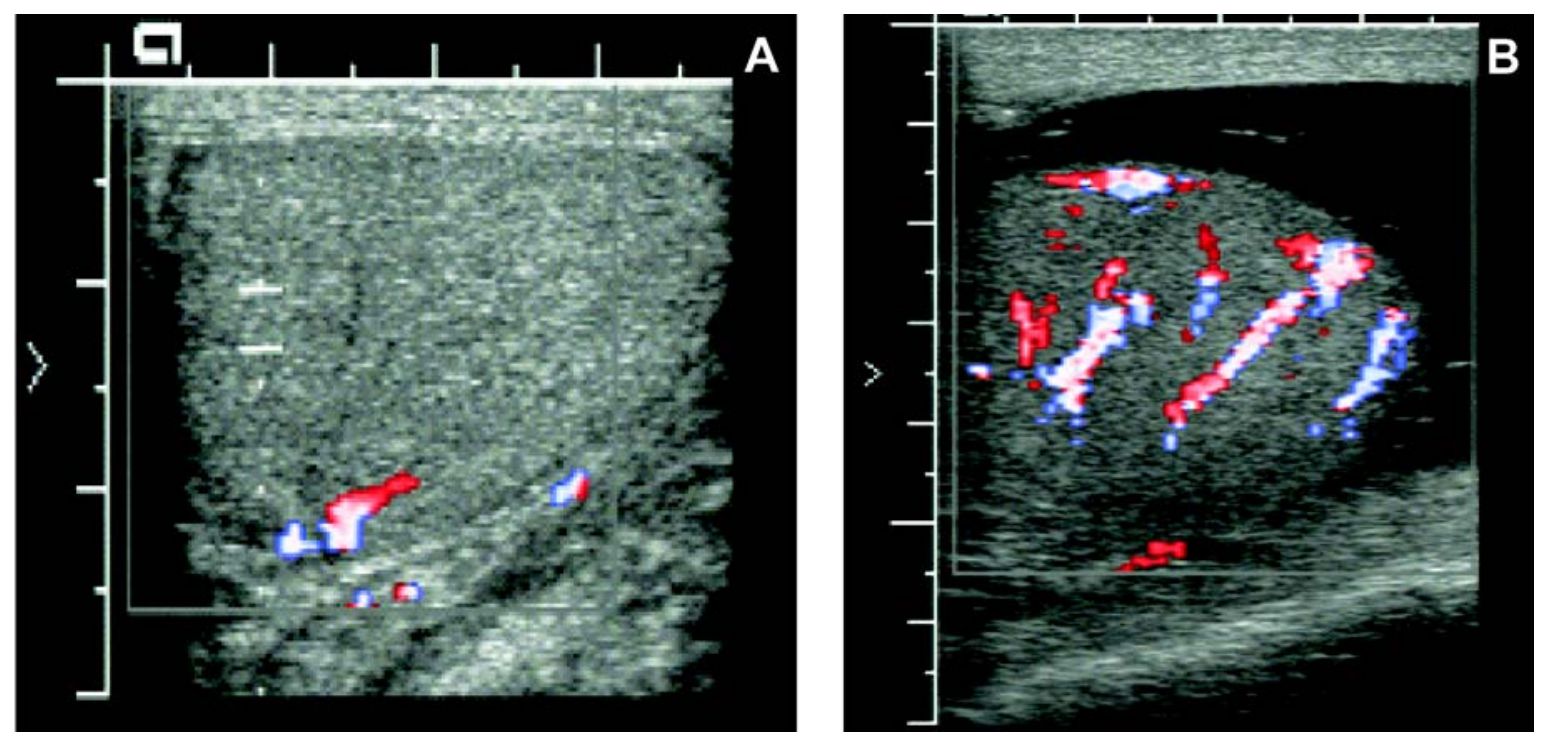

Figure 1 - Testicular ultrasound showing a normal blood flow in the right testicle (A) and increased blood flow in the left testicle (B).

melitensis, B. uis or B. canis. Brucellosis may cause granulomatous orchitis in up to $20 \%$ of affected patients (1). Patients usually present with acute symptoms of less than 2 weeks of duration (1). Although patients may have unilateral epididymoorchitis or orchitis alone, bilateral epididymo-orchitis occurs in up to $59 \%$ of affected patients (1) and may occur in the absence of systemic symptoms in endemic areas (2).

Brucella epididymo-orchitis can be distinguished from nonspecific epididymo-orchitis by a history of contact with animals, consuming raw milk or cheese made from it, gradual onset, longer duration, typical undulant fever, relatively minimal local
A

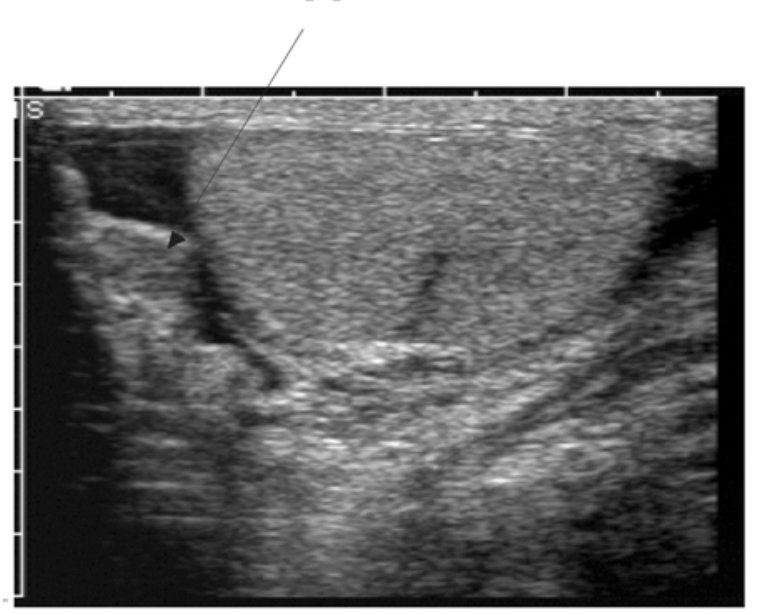

B

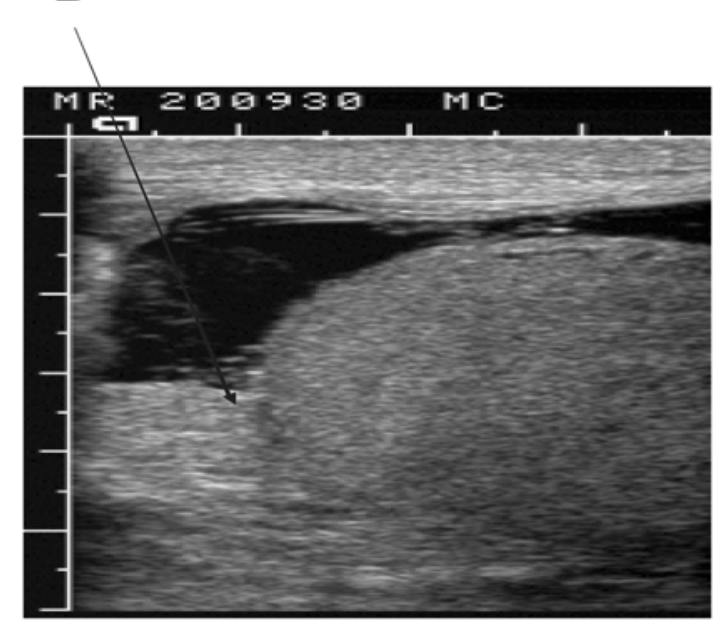

Figure 2 - Testicular ultrasound showing a normal right epididymis (A) and an enlarged and thickened left epididymis (B). 
inflammation, and absence of lower urinary tract symptoms and no significant leukocytosis (1). The differentiation is important since the delay of specific treatment increases the risk of contralateral involvement, necrosis and systemic manifestations (3). Thus, in endemic areas, the suspicion is enough to initiate therapy for brucellosis while awaiting the confirmatory laboratory tests (1).

As in our case, normal urine analysis and culture is reported in the majority of patients with Brucella epididymo-orchitis (65\%) (1). The majority of patients with Brucella epididymo-orchitis have initial agglutination titers of $>1: 320,53-69 \%$ of patients have positive blood cultures (1) and 6.7\% have positive culture from epididymal aspirations. Doxycycline plus rifampicin for 6 weeks or doxycycline (6 weeks) plus gentamicin for 2-3 weeks is usually prescribed for treatment. Alternatively, streptomycin intramuscularly and tetracycline, with or without cotrimoxazole orally may be used in cases that do not respond to standard therapy. The complication rate is usually low with $5 \%$ of patients developing necrotizing orchitis requiring orchiectomy.

In conclusion, brucella epididymo-orchitis should be a consideration in the differential diagnosis of patients presenting with signs and symptoms of this entity in endemic areas of brucellosis. The condition usually has a favorable outcome with the standard treatment of brucellosis. Inappropriate management of Brucella epididymo-orchitis may result in serious complications such as testicular abscess, atrophy and male infertility.

\section{ACKNOWLEDGMENT}

The author acknowledges the use of Saudi Aramco Medical Services Organization (SAMSO) facilities for the data and study, which resulted in this paper. Opinions expressed in this article are those of the author and not necessarily of SAMSO.

\section{CONFLICT OF INTEREST}

None declared.

\section{REFERENCES}

1. Papatsoris AG, Mpadra FA, Karamouzis MV, Frangides CY: Endemic brucellar epididymo-orchitis: a 10-year experience. Int J Infect Dis. 2002; 6: 309-13.

2. Kocak I, Dundar M, Culhaci N, Unsal A: Relapse of brucellosis simulating testis tumor. Int J Urol. 2004; 11: 683-5.

3. Alapont Alacreu JM, Gomez Lopez L, Delgado F, Palmero Marti JL, Pacheco Bru JJ, Pontones Moreno JL, et al.: Brucellar orchiepididymitis. Actas Urol Esp. 2004; 28: 774-6.

\footnotetext{
Correspondence address:

Dr. Jaffar A. Al-Tawfiq

PO Box 76, Room A-420B, Building 61

Dhahran Health Ctr, Saudi Aramco

Dhahran 31311, Saudi Arabia

Fax: + 9663 877-3790

E-mail: jaffar.tawfiq@aramco.com
}

Accepted after revision: September 30, 2005 